\title{
COVID-19 e disfagia: guia prático para atendimento hospitalar seguro - número 1
}

\section{COVID-19 and dysphagia: practical guide to safe hospital care -}

\section{number 1}

\author{
Brenda Carla Lima Araújo' (D), Danielle Ramos Domenis ${ }^{1}$ (D), Thiago Henrique de Pontes Ferreira ${ }^{2}$ (D), \\ Christiane Lopes de Albuquerque Merelles ${ }^{3}$ (D), Thales Rafael Correia de Melo Lima ${ }^{4}$ (1)
}

\section{RESUMO}

A pandemia da COVID-19 é associada com o novo tipo do coronavírus, o SARS-Cov-2, que causa síndrome respiratória aguda grave. Esse vírus afeta o trato respiratório superior e é transmitido, principalmente, por gotículas, espirros e aerossóis e, por isso, há um alto risco de transmissão durante os procedimentos do fonoaudiólogo que atua no ambiente hospitalar. O objetivo deste artigo foi descrever as iniciativas que podem ser adotadas pelo fonoaudiólogo que atende pacientes à beira do leito, com disfagia, visando diminuir o risco de contaminação cruzada na prática clínica durante a pandemia do SARS-CoV-2.

Palavras-chave: Coronavírus; transtorno de deglutição; Covid-19; fonoaudiologia; infecção por coronavírus.

\begin{abstract}
The COVID-19 pandemic is associated with a new strain of coronavirus, SARS-Cov-2, which causes severe acute respiratory syndrome. This virus affects the upper respiratory tract and is transmitted mainly by droplets, sneezes and aerosols, so there is a high risk of transmission during the procedures of the speech therapist who works in the hospital environment. This article aims to describe the steps that can be taken by the speech therapist who attends bedside patients with dysphagia, to reduce the risk of cross-contamination in clinical practice during the COVID-19 pandemic.
\end{abstract}

Keywords: Coronavirus; dysphagia; Covid-19; speech therapy; coronavirus infection

Trabalho realizado no Departamento de Fonoaudiologia, Universidade Federal de Sergipe - UFS - São Cristóvão (SE), Brasil.

${ }^{1}$ Departamento de Fonoaudiologia, Universidade Federal de Sergipe - UFS - São Cristóvão/Lagarto (SE), Brasil.

${ }^{2}$ Instituto Universitário Italiano de Rosário - IUNIR - Rosário (SF), Argentina.

${ }^{3}$ Serviço de Fonoaudiologia, Universidade do Estado do Rio de Janeiro - UERJ - Rio de Janeiro (RJ), Brasil

${ }^{4}$ Programa de Pós-graduação Profissional em Gestão e Inovação Tecnológica em Saúde, Universidade Federal de Sergipe - UFS - Aracaju (SE), Brasil.

Conflito de interesses: Não.

Contribuição dos autores: BCLA participou da idealização do estudo, coleta, análise e interpretação dos dados e redação do artigo; DRD, THPS e CLAM participaram da redação final do artigo; TRCM participou da idealização do estudo, análise, interpretação dos dados e redação do artigo.

Financiamento: Nada a declarar.

Autor correspondente: Brenda Carla Lima Araújo. E-mail: brendaaraujo@yahoo.com.br

Recebido em: Julho 13, 2020; Aceito: Setembro 24, 2020. 
O coronavírus é um dos principais patógenos que têm como alvo o sistema respiratório humano. Existem relatos na literatura sobre a transmissão humana do coronavírus em ambientes de saúde e domiciliares ${ }^{(1)}$. Também há evidências de que o vírus se espalha, principalmente, por espirros, gotículas de saliva e aerossóis ${ }^{(2,3)}$. O sintoma comum da COVID-19 em pacientes críticos é a síndrome do desconforto respiratório agudo e a maioria dos pacientes hospitalizados requer suporte respiratório ${ }^{(4)}$. Estudo recente ${ }^{(5)}$ sobre desempenho e segurança da deglutição em pacientes com COVID-19 encontrou, durante a primeira avaliação da deglutição, $19,8 \%$ dos pacientes nos níveis 1-3 da escala ASHA (quando o indivíduo é incapaz de engolir com segurança por via oral e uma via de alimentação alternativa é necessária) e 53,5\% dos pacientes deglutiram com segurança, porém, com restrição alimentar e uso de manobras compensatórias. Por outro lado, o estudo também sugeriu menos sessões de reabilitação para retorno à alimentação oral segura, em comparação aos pacientes críticos internados em unidade de terapia intensiva (UTI), sem COVID-19, além de permanecerem intubados por mais tempo.

O ritmo da literatura emergente da COVID-19 e atualizações diárias das políticas nacionais de saúde e recomendações resultaram em uma mudança rápida sobre os temas com relevância para essa doença. Nesse contexto, considerando as evidências sobre o alto risco de transmissão do SARS-CoV-2 durante os procedimentos do fonoaudiólogo inserido em ambiente hospitalar, este artigo teve como objetivo fornecer as evidências científicas atuais do manejo seguro do fonoaudiólogo à beira do leito, durante a atual pandemia da COVID-19.

\section{Equipamento de proteção individual - EPI}

- Os fonoaudiólogos devem priorizar o uso de equipamentos de proteção individual, como respirador N95 ou PFF2 (sempre verificar a vedação, ao colocar a máscara), roupa privativa do hospital, luvas de procedimento, óculos para proteção, protetor facial de acrílico, touca e aventais descartáveis. Higienizar as mãos com água e sabão, conforme as medidas de precaução padrão, ou esfregar as mãos com etanol $70 \%$ ou isopropanol a $70 \%{ }^{(6)}$, antes e após o contato com o paciente, bem como após a retirada do EPI e nas superfícies ambientais circundantes. Estudo demonstrou que o SARS-CoV pode ser inativado facilmente com desinfetantes comumente usados $^{(7)}$. Além disso, também há evidência de que esfregar as mãos com etanol ou isopropanol, ambos $70 \%$, é, geralmente, eficaz contra vírus envelopados, incluindo SARS-CoV e MERS-CoV ${ }^{(8)}$.

\section{Fonoaudiologia e COVID-19}

- O atendimento fonoaudiológico em pacientes com exame negativo para COVID-19 deve ser realizado com EPIs apropriados. No caso de pacientes com suspeita ou confirmação de COVID-19, o atendimento fonoaudiológico não é recomendado, devido ao risco de geração de aerossol $l^{(9)}$. No entanto, ainda existem controvérsias na literatura. Sugere-se que, durante o curso da doença, o atendimento seja indicado apenas em casos de necessidade absoluta por deficit significativo da deglutição, quando o adiamento da terapia impõe maior risco de agravo à saúde. Além disso, o atendimento, nesses casos, deve priorizar medidas que gerem o mínimo possível de aerossóis, após acordo da equipe e com utilização de todos os EPIs adequados. É necessário definir critérios para solicitação do serviço de fonoaudiologia, além de orientação de práticas seguras para a assistência fonoaudiológica hospitalar ${ }^{(10,11)}$. Deve-se privilegiar medidas mínimas de manuseio (procedimentos de adaptação, compensações, orientações e monitoramento da deglutição) e analisar a relevância da avaliação fonoaudiológica diante do quadro clínico do paciente. Pacientes com história de risco de broncoaspiração são elegíveis para avaliação fonoaudiológica, como: idoso frágil, doença neurológica, câncer de cabeça e pescoço, doença pulmonar crônica, intubação orotraqueal maior que 48 horas e traqueostomia ${ }^{(12,13)}$.

\section{Exame clínico para pacientes com disfagia}

- A inspeção oral e o manuseio da boca, língua e mucosa oral devem ser evitados; caso sejam necessários, devese garantir a proteção adequada de toda a face e os demais cuidados; recomenda-se evitar qualquer tipo de estímulo que possa desencadear reflexos de tosse e vômito, inclusive em áreas da cavidade oral, como a base da língua, fauces, úvula, palato e parede da faringe ${ }^{(14)}$. Estudo mostrou carga viral elevada na saliva de pacientes infectados, após 25 dias dos primeiros sintomas ${ }^{(14)}$. Essas formas de transmissão são importantes para os devidos cuidados na prática fonoaudiológica hospitalar, uma vez que muitos procedimentos realizados pelo fonoaudiólogo requerem a proximidade com a face do paciente, além do contato com a mucosa oral e fluidos corporais, como saliva e gotículas respiratórias ${ }^{(15)}$.

- Sugere-se realizar a avaliação da deglutição com múltiplas consistências e utensílios descartáveis, para definição da via de nutrição mais segura e adequada, considerando a história prévia, a doença atual e o desempenho orofaríngeo, com oferta e monitoramento pela equipe de enfermagem, se for o caso, para redução de múltiplas visitas. Nos casos de extubação, a avaliação pode ser realizada após 48 horas $^{(10)}$ Recomenda-se descartar e não reutilizar as sobras de alimentos (com ou sem espessantes) utilizadas na avaliação da deglutição. Estudo sugeriu que o SARS-CoV pode ser transmitido indiretamente, por meio do contato da mucosa, de fonte de água e alimentos poluídos ${ }^{(16)}$.

- Evitar realização da ausculta cervical e, se necessário, utilizar apenas o estetoscópio disponível no leito do paciente. O estetoscópio gera a possibilidade de propagação do vírus e contaminação de dispositivos hospitalares, podendo causar o contágio de profissionais de saúde e de pacientes hospitalizados ${ }^{(17)}$. E importante considerar a limpeza e desinfecção das superfícies dos equipamentos que foram utilizados no atendimento ao paciente, usando-se germicidas hospitalares. Até o momento, o que se sabe sobre a sobrevivência do SARS-CoV-2 é que ele é mais estável em superfícies de plástico e aço inoxidável ${ }^{(18)}$. 


\section{Cuidados com a traqueostomia e COVID-19}

- Em casos de pacientes traqueostomizados e procedimentos relacionados, como aspiração endotraqueal e desinsuflação do balonete de cuff, é recomendado evitar este tipo de conduta devido à grande chance de produção de tosse e "gotículas" de fluido do trato respiratório, por serem potencialmente geradores de aerossóis ${ }^{(19)}$. Em estudo de revisão sistemática, a traqueostomia foi considerada um dos procedimentos que mais gera aerossóis ${ }^{(20)}$. Recomendam-se cânulas sem fenestra e com cuff, que deve permanecer insuflado, até que o teste para COVID-19 seja negativo ${ }^{(21)}$. No entanto, sugere-se uma avaliação individual do paciente. A desinsuflação do cuff e procedimentos relacionados à traqueostomia devem ocorrer apenas em sistema fechado de aspiração e, em casos de absoluta necessidade, realizados após o período de quarentena e negativação da doença. As trocas e limpeza das cânulas de traqueostomias devem ser evitadas, a menos que sejam consideradas urgentes. O uso de válvulas de fala deve ser discutido com a equipe multiprofissional ${ }^{(22)}$. Riscos e benefícios devem ser avaliados, levando-se em consideração os benefícios para o manejo das vias aéreas, fonação e fisiologia da deglutição. Estudos evidenciaram que, durante um espirro ou tosse, esses fluidos do trato respiratório com carga viral, geralmente maior que $5 \mathrm{~mm}$ de diâmetro, podem ser inalados através de partículas microscópicas de aerossol ${ }^{(23)}$. Outro estudo sugeriu que o SARS-CoV-2 pode ser transmitido pelo ar, por meio de aerossóis formados durante procedimentos médicos ${ }^{(24)}$. Porém, a via de transmissão do aerossol e as vias de transmissão pelas fezes e pela boca ainda precisam ser esclarecidas ${ }^{(24)}$. Ainda não há evidências que sustentem a terapia fonoaudiológica em pacientes traqueostomizados infectados, além dos reais impactos desses mecanismos na transmissão do SARS-CoV-2.

\section{Avaliação complementar da deglutição e outras estratégias}

- Exames complementares para avaliação da deglutição e voz devem ser evitados ${ }^{(25)}$. As evidências sugerem que a avaliação endoscópica flexível e, provavelmente, a videofluoroscopia da deglutição, apresentam alto risco de contaminação para pacientes, fonoaudiólogos e otorrinolaringologistas, além da equipe envolvida no procedimento ou reprocessamento do equipamento ${ }^{(26)}$.

- No cenário atual, deve-se considerar o atendimento remoto como uma alternativa de prestação de serviços, de forma complementar, ou em substituição aos tratamentos presenciais. Estudo ${ }^{(27)}$ enfatizou que a abordagem para avaliar a disfagia deve ser adaptada, com objetivo de reduzir o risco de possível exposição viral. A história clínica e a avaliação dos pacientes de forma remota, além do uso de questionários validados, podem auxiliar na triagem e seleção de pacientes que necessitam de avaliação e diagnóstico presencial. Por outro lado, ainda há uma lacuna sobre a eficácia da telerreabilitação em pacientes disfágicos, quando comparada à terapia presencial ${ }^{(28)}$. A Figura 1 apresenta um fluxograma com o guia prático para cuidados seguros no hospital, durante a pandemia da COVID-19.

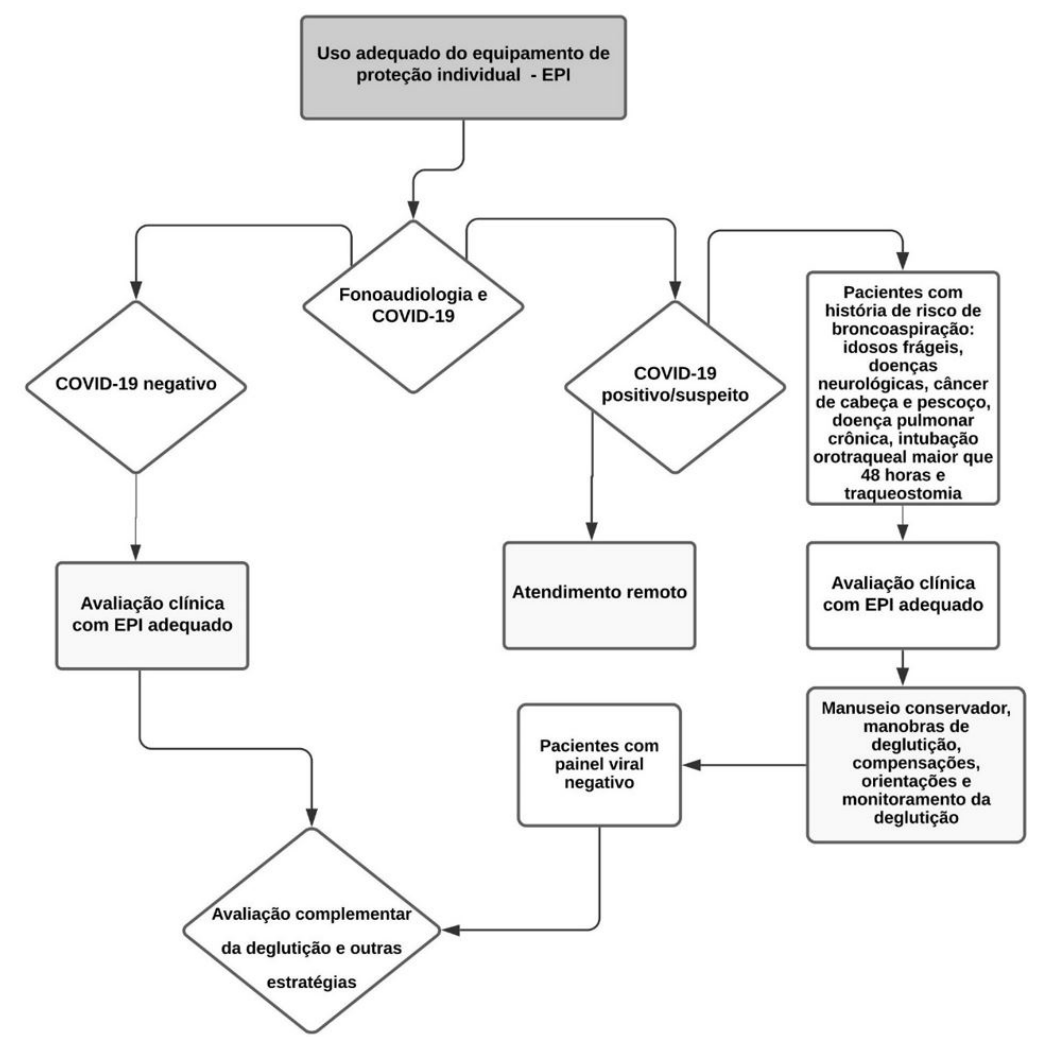

Figura 1. Guia prático para atendimento hospitalar seguro na COVID-19 
Por fim, as medidas de precaução padrão devem ser mantidas para todos os pacientes sem sintomas. Recomenda-se a utilização de protocolos de biossegurança e as medidas de proteção específicas devem ser rigorosamente seguidas durante os atendimentos fonoaudiológicos em ambiente hospitalar. Além disso, sugerem-se diretrizes práticas, como triagem e avaliação de pacientes disfágicos, mediante protocolos validados e adequados para atendimento fonoaudiológico hospitalar em pacientes com COVID-19.

\section{REFERÊNCIAS}

1. Chan JFW, Yuan S, Kok KH, To KKW, Chu H, Yang J, et al. A familial cluster of pneumonia associated with the 2019 novel coronavirus indicating person-to-person transmission: A study of a family cluster. Lancet. 2020;395(10223):514-23. http://dx.doi.org/10.1016/S01406736(20)30154-9. PMid:31986261.

2. Lu CW, Liu XF, Jia ZF. 2019-nCoV transmission through the ocular surface must not be ignored. Lancet. 2020;395(10224):e39. http:// dx.doi.org/10.1016/S0140-6736(20)30313-5. PMid:32035510.

3. Peng X, Xu X, Li Y, Cheng L, Zhou X, Ren B. Transmission routes of 2019-nCoV and controls in dental practice. Int J Oral Sci. 2020;12(1):16. http://dx.doi.org/10.1038/s41368-020-0075-9. PMid:32127517.

4. Mohan R, Mohapatra B. Shedding light on Dysphagia associated with COVID-19: The what and why. OTO Open. 2020;4(2):1-2.

5. Lima MS, Sassi FC, Medeiros GC, Ritto AP, Andrade CRF. Preliminary results of a clinical study to evaluate the performance and safety of swallowing in critical patients with COVID-19. Clinics. 2020;75(8):e2021. http://dx.doi.org/10.6061/clinics/2020/e2021. PMid:32555948.

6. Centers for Disease Control and Prevention. CDC Statement for Healthcare Personnel on Hand Hygiene during the Response to the International Emergence of COVID-19 [Internet]. Atlanta: CDC; 2020 [cited 2020 Apr 24]. Available from: https://www.cdc.gov/coronavirus/2019-ncov/ hcp/hand-hygiene.html?CDC_AA_refVal=https $\% 3 \mathrm{~A} \% 2 \mathrm{~F} \% 2 \mathrm{Fwww}$. cdc.gov\%2Fcoronavirus\%2F2019-ncov\%2Finfection-control\%2Fhcphand-sanitizer.html

7. Rabenau HF, Kampf G, Cinatl J, Doerr HW. Efficacy of various disinfectants against SARS coronavirus. J Hosp Infect. 2005;61(2):10711. http://dx.doi.org/10.1016/j.jhin.2004.12.023. PMid:15923059.

8. Kampf G. Efficacy of ethanol against viruses in hand disinfection. J Hosp Infect. 2018;98(4):331-8. http://dx.doi.org/10.1016/j.jhin.2017.08.025. PMid:28882643.

9. Kimura Y, Ueha R, Furukawa T, Oshima F, Fujitani J, Nakajima J. Society of swallowing and dysphagia of Japan: Position statement on dysphagia management during the COVID-19 outbreak. Auris Nasus Larynx. 2020;47(5):715-726. http://dx.doi.org/10.1016/j. anl.2020.07.009.

10. AMIB: Associação de Medicina Intensiva Brasileira. Parecer do Departamento de Fonoaudiologia da AMIB referente ao atendimento ao COVID19 na terapia intensiva e no ambiente hospitalar. Vila Olímpia: AMIB; 2020 [cited 2020 Apr 24]. Available from: https:// www.amib.org.br/fileadmin/user_upload/amib/2020/marco/22/14 Parecer_FonoaudiologiaCOVID-19.pdf

11. ASHA: American Speech-Language-Hearing Association. SLP Service Delivery Considerations in Health Care During Coronavirus/ COVID-19. Rockville, MD: ASHA; 2020 [cited 2020 Apr 24]. Available from: https://www.asha.org/SLP/healthcare/SLP-Service-DeliveryConsiderationsin-Health-Care-During-Coronavirus/
12. Igarashi K, Kikutani T, Tamura F. Survey of suspected dysphagia prevalence in home-dwelling older people using the 10-Item Eating Assessment Tool (EAT-10). PLoS ONE. 2019;14(1):1-13. https://doi. org/10.1371/journal.pone.0211040.

13. Zuercher P, Moret CS, Dziewas R, Schefold JC. Dysphagia in the intensive care unit: Epidemiology, mechanisms, and clinical management. Crit Care. 2019;23(103):1-11. http://dx.doi.org/10.1186/s13054-0192400-2. PMid:30922363.

14. To KKW, Tsang OTY, Leung WS, Tam AR, Wu TC, Lung DC, et al. Temporal profiles of viral load in posterior oropharyngeal saliva samples and serum antibody responses during infection by SARS-CoV-2: an observational cohort study. Lancet Infect Dis. 2020;20(5):565-74. http://dx.doi.org/10.1016/S1473-3099(20)30196-1. PMid:32213337.

15. Speech Pathology Association of Australia. Speech Pathology Australia guidance for service delivery, clinical procedures and infection control during COVID-19 pandemic. Melbourne, Australia: SPA; 2020.

16. Ceylan Z, Meral R, Cetinkaya T. Relevance of SARS-CoV-2 in food safety and food hygiene: potential preventive measures, suggestions and nanotechnological approaches. VirusDis. 2020;31(2):154-60. http://dx.doi.org/10.1007/s13337-020-00611-0. PMid:32656309.

17. Buonsenso D, Pata D, Chiaretti A. COVID-19 outbreak: less stethoscope, more ultrasound. Lancet Respir Med. 2020;2600(20):30120. http:// dx.doi.org/10.1016/S2213-2600(20)30120-X. PMid:32203708.

18. van Doremalen N, Bushmaker T, Morris DH, Holbrook MG, Gamble A, Williamson BN, et al. Aerosol and Surface Stability of SARS-CoV-2 as Compared with SARS-CoV-1. N Engl J Med. 2020;382(16):1564-7. http://dx.doi.org/10.1056/NEJMc2004973. PMid:32182409.

19. Zaga CJ, Pandian V, Brodsky MB, Wallace S, Cameron TS, Chao $\mathrm{C}$, et al. Speech-language pathology guidance for tracheostomy during the COVID-19 Pandemic: An international multidisciplinary perspective. Am J Speech Lang Pathol. 2020;29(3):1-15. http://dx.doi. org/10.1044/2020_AJSLP-20-00089. PMid:32525695.

20. Tran K, Cimon K, Severn M, Pessoa-Silva CL, Conly J. Aerosol generating procedures and risk of transmission of acute respiratory infections to healthcare workers: A systematic review. PLoS One. 2012;7(4):e35797. http://dx.doi.org/10.1371/journal.pone.0035797. PMid:22563403.

21. Piccin O, Albertini R, Caliceti U, Cavicchi O, Cioccoloni E, Demattè $\mathrm{M}$, et al. Early experience in tracheostomy and tracheostomy tube management in Covid-19 patients. American Journal of Otolaryngology. 2020;41(4):102535. https://doi.org/10.1016/j.amjoto.2020.102535

22. Namasivayam-Macdonald AM, Riquelme LF. Speech-language pathology management for adults with COVID-19 in the acute hospital setting: Initial recommendations to guide clinical practice. Am J Speech Lang Pathol. 2020;17:1-16. http://dx.doi.org/10.1044/2020_AJSLP-20-00096. PMid:32692584.

23. Ghinai I, McPherson TD, Hunter JC, Kirking HL, Christiansen D, Joshi K, et al. First known person-to-person transmission of severe acute respiratory syndrome coronavirus 2 (SARS-CoV-2) in the USA. Lancet. 2020;395(10230):1137-44. http://dx.doi.org/10.1016/S01406736(20)30607-3. PMid:32178768.

24. Wax RS, Christian MD. Practical recommendations for critical care and anesthesiology teams caring for novel coronavirus (2019-nCoV) patients. Can J Anesth. 2020;67(5):568-76. http://dx.doi.org/10.1007/ s12630-020-01591-x. PMid:32052373.

25. Rameau A, Young VVN, Amin MR, Sulica L. Flexible Laryngoscopy and COVID-19. Otolaryngol. Head Neck Surg. 2020; 162(6):813-815.

26. Mattei A, Amy de la Bretèque B, Crestani S, Crevier-Buchman L, Galant C, Hans S, et al. Guidelines of clinical practice for the management of swallowing disorders and recent dysphonia in the context of the 
COVID-19 pandemic. Eur. Ann. Otorhinolaryngol. Head Neck Dis 2020;137(3):173-175

27. Soldatova L, Williams C, Postma GN, Falk GW, Mirza N. Virtual dysphagia evaluation: practical guidelines for dysphagia management in the context of the COVID-19 pandemic. Otolaryngol Head Neck
Surg. 2020;163(3):1-4. http://dx.doi.org/10.1177/0194599820931791 PMid:32450732.

28. Nordio S, Innocenti T, Agostini M, Meneghello F, Battel I. The efficacy of telerehabilitation in dysphagic patients: a systematic review. Acta Otorhinolaryngol Ital. 2018;38(2):79-85. PMid:29967554. 